\title{
Ecological analysis of ethnic differences in relation between tuberculosis and poverty
}

\author{
Jeremy I Hawker, Surinder S Bakhshi, Shaukat Ali, C Paddy Farrington
}

\begin{abstract}
Objective To examine the effect of ethnicity on the relation between tuberculosis and deprivation. Design Retrospective ecological study comparing incidence of tuberculosis in white and south Asian residents of the 39 electoral wards in Birmingham with ethnic specific indices of deprivation.

Setting Birmingham, 1989-93.

Subjects 1516 notified cases of tuberculosis. Main outcome measures Rates of tuberculosis and measures of deprivation.

Results Univariate analysis showed significant associations of tuberculosis rates for the whole population with several indices of deprivation $(\mathrm{P}<0.01)$ and with the proportion of the population of south Asian origin $(\mathrm{P}<0.01)$. All deprivation covariates were positively associated with each other but on multiple regression, higher level of overcrowding was independently associated with tuberculosis rates. For the white population, overcrowding was associated with tuberculosis rates independently of other variables $(\mathrm{P}=0.0036)$. No relation with deprivation was found for the south Asian population in either single or multivariable analyses.
\end{abstract}

Conclusions Poverty is significantly associated with tuberculosis in the white population, but no such relation exists for those of Asian ethnicity. These findings suggest that causal factors, and therefore potential interventions, will also differ by ethnic group.

\section{Introduction}

Tuberculosis has been linked with poverty since the 19th century. ${ }^{1}$ Notification rates for tuberculosis have fallen substantially in England and Wales this century, ${ }^{2}$ coinciding with improving living conditions and improved treatment. However, since the 1960s this decline has slowed, with an increasing proportion of cases occurring in immigrants from countries with a high prevalence of tuberculosis. ${ }^{2}$ The proportion of notified cases in the United Kingdom among people of south Asian origin (hereafter termed Asians) has increased successively in the six national surveys carried out between 1965 and 1993 to $41 \%$ of cases, whereas the proportion in white people has fallen to $44 \%{ }^{3}$

Recent ecological studies in Liverpool and elderly people in Leeds suggest that tuberculosis is associated with various indices of deprivation. ${ }^{4}$ However, neither of these studies was able to analyse this relation by ethnic group. Disentangling the effects of deprivation from those of belonging to an ethnic minority on the incidence of tuberculosis is difficult, ${ }^{6}$ but to attempt to do so is important. The epidemiology of tuberculosis differs considerably with ethnicity. Asian people predominantly acquire new infection from infected people in the same community or when visiting the
Indian subcontinent, ${ }^{7}$ but white people generally have reactivation of endogenous latent infection. ${ }^{8}$ This distinction is reflected in the differences in the age distribution of cases and in the type of disease between ethnic groups. In the 1988 national survey, 55\% of white people with tuberculosis were over 55 years old compared with only $16 \%$ of Asians, and $16 \%$ of white patients had only non-respiratory tuberculosis compared with $36 \%$ of Asian patients. ${ }^{9}$ Indeed, age is an important risk factor affecting the magnitude of tuberculosis morbidity in the population, ${ }^{10}$ but the age distribution of the population of these two ethnic groups differs considerably. It is therefore not safe to assume that social factors which affect tuberculosis incidence in one of these ethnic groups will do so for the other. We attempted to discover whether the link between tuberculosis and deprivation holds true in Birmingham for the whole population and for white and Asian ethnic groups analysed separately.

\section{Methods}

We obtained details of notifications of tuberculosis (excluding chemoprophylaxis) in Birmingham for the five years 1989-93 from the Birmingham tuberculosis notification register. Patients are asked to indicate their ethnic group (Asian, white, black Caribbean, or other), which is recorded on the tuberculosis register by staff at the Birmingham chest clinic. The electoral ward of residence is obtained from the recorded home address.

We used 1991 census data to provide denominators to calculate specific mean annual notification rates per 100000 population for each ethnic group in each of the 39 electoral wards in Birmingham. We added 0.5 to each ward count of tuberculosis cases to reduce biases associated with zero counts. The census data were also used to obtain the following indices of deprivation: proportion of households with more than 1.5 people per room; proportion of households with more than one person per room; proportion of residents in households with more than 1.5 people per room; proportion of households not owner occupied; proportion of residents in households lacking central heating; proportion of households with no car; proportion of residents in households with no car; proportion of residents unemployed. These covariates were calculated separately for white and Asian residents and households in which the head of the household was white or Asian. Other covariates used were the Townsend score, which is derived from four of these variables, ${ }^{11}$ and, for the analysis of total population tuberculosis, the proportion of the population of Asian ethnicity.

We used Box-Cox profiles ${ }^{12}$ to assess the need for transformations of the rates. In all cases the square root transformation was found adequate. Wards were weighted according to ward populations. For regressions involving only Asians or white people, the Asian
Public Health

Laboratory Service Communicable Disease

Surveillance Centre (West Midlands),

Birmingham

Heartlands

Hospital, Bordesley Green East,

Birmingham B9 5SS

Jeremy I Hawker consultant

epidemiologist

Birmingham Health Authority, St Chads Court, Birmingham B16 9RG

Surinder S Bakhshi consultant in

communicable disease control

Shaukat Ali research associate

Department of Statistics, Open University, Walton

Hall, Milton Keynes MK7 6AA

C Paddy Farrington senior statistician

Correspondence to: J I Hawker jhawker@cdscwmid. demon.co.uk

BMJ 1999;319:1031-4 
Table 1 Tuberculosis cases by ethnic group and age group

\begin{tabular}{lcc} 
& \multicolumn{2}{c}{ No (\%) of cases } \\
\cline { 2 - 3 } Age (years) & White & Asian \\
\hline $0-15$ & $39(12)$ & $101(10)$ \\
\hline $16-34$ & $54(16)$ & $379(38)$ \\
\hline $35-64$ & $132(40)$ & $386(39)$ \\
\hline$\geqslant 65$ & $107(32)$ & $129(13)$ \\
\hline Total & 332 & 995 \\
\hline
\end{tabular}

or white ward populations, respectively, were used. We used S-plus software for multiple weighted linear regression of the square roots of the tuberculosis rates against predictor variables and diagnostic checks (using residual plots) of the validity of the model..$^{13}$

Table 2 Parameter estimates (SE) of overcrowding and proportion of Asian residents for whole population with four regression models

\begin{tabular}{lcccc} 
& \multicolumn{4}{c}{ Regression model } \\
\cline { 2 - 5 } & (a) & (b) & (c) & (d) \\
\hline Intercept & $2.58(0.46)$ & $2.12(0.60)$ & $3.69(0.64)$ & $3.85(0.68)$ \\
\hline \% households with $>1.5$ people/room & $2.33(0.34)$ & - & $5.45(1.38)$ & $5.18(1.44)$ \\
\hline \% households with $>1$ person/room & - & $0.603(0.11)$ & $-0.91(0.39)$ & $-1.02(0.42)$ \\
\hline \% Asians residents & - & - & - & $0.04(0.055)$ \\
\hline
\end{tabular}
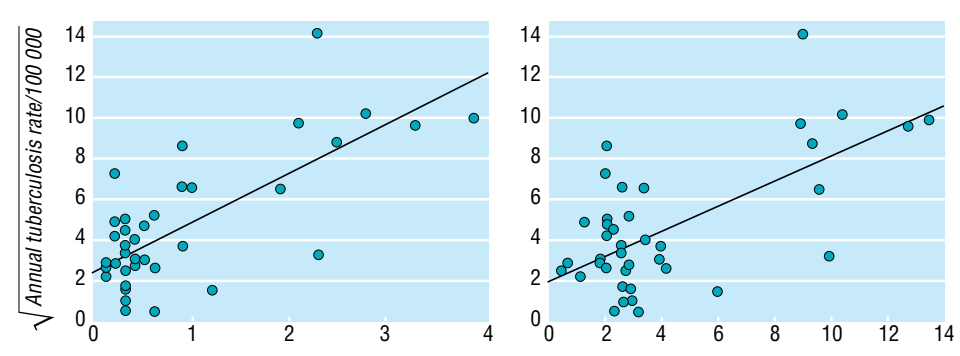

Households with $>1.5$ people/room (\%)

Households with $>1$ person/room (\%)

Fig 1 Relation between tuberculosis rate and overcrowding for whole population. Each point represents one electoral ward

Table 3 Median values for tuberculosis rate and selected covariates in 39 electoral wards by ethnic group and overall population

\begin{tabular}{lccc} 
& Whites & Asians & Overall \\
\hline Annual rate/100 000 & 7.90 & 110.1 & 13.76 \\
\hline$\%$ households $>1.5$ people/room & 0.32 & 3.7 & 0.40 \\
\hline$\%$ unemployed & 12 & 16 & 14 \\
\hline$\%$ households without car & 44 & 24 & 44 \\
\hline$\%$ households not owner occupied & 40 & 18 & 42 \\
\hline
\end{tabular}
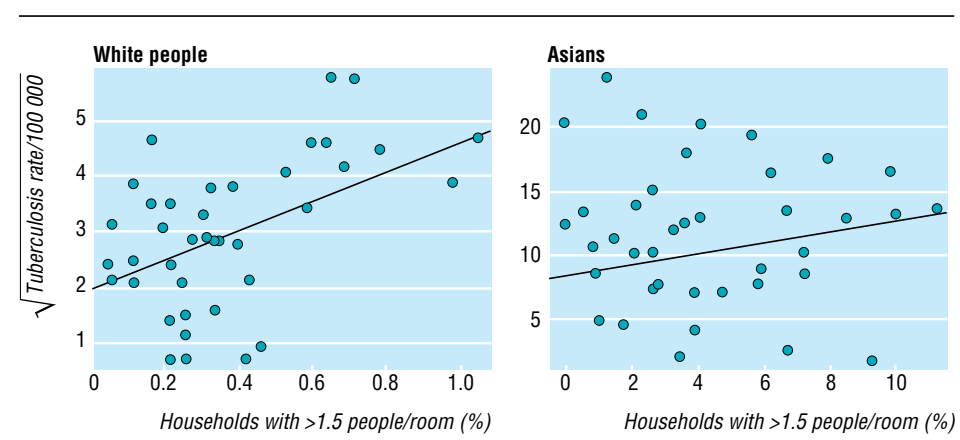

Fig 2 Relation between tuberculosis rate and overcrowding by ethnic group. Figures are annual averages and each point represents one electoral ward

\section{Results}

During the study period, 1516 cases of tuberculosis were notified in Birmingham, of which 995 (66\%) were in Asians and $332(22 \%)$ in white people. The crude annual notification rates were $153 / 100000$ population for Asian people and 8.8/100 000 for white people, a 17 -fold difference. Table 1 gives the age distribution of cases in the two ethnic groups.

\section{Total population analysis}

In single variable analyses all but two of the 10 covariates were strongly positively associated with tuberculosis $(\mathrm{P}<0.01$ in each case). The exceptions were the proportion of households not owning their own homes $(\mathrm{P}=0.09)$ and the proportion of residents without central heating $(\mathrm{P}=0.069)$. However, all these covariates were positively correlated. In multiple regression the only variables independently associated with tuberculosis were the two overcrowding variables. These two variables were themselves dependent. Table 2 shows three models for overcrowding (models (a)-(c)). Model (c) shows that when both overcrowding variables are fitted together, one has a negative coefficient that partly compensates for the other. Overall the models indicate a positive relation with high levels of overcrowding (fig 1). The proportion of Asian residents was not independently associated with tuberculosis (table 2(d)).

However, the differences in both tuberculosis rates and deprivation indices between the Asian and white community, shown in table 3 and figure 2, throw doubt on the validity of such grouped analyses. Specifically, the relation between deprivation and tuberculosis at the individual level may be confounded by ethnicity. Figure 3 shows that the level of overcrowding is strongly correlated with the proportion of the population of Asian ethnicity.

\section{Analysis by ethnic group}

Figure 2 shows the tuberculosis rates plotted against the proportions of households with more than 1.5 people per room for the white and Asian populations. In single variable analyses for white residents the only variables significantly associated with tuberculosis rates were the proportion of households with more than 1.5 people per room $(\mathrm{P}=0.0036)$ and the proportions of residents in such households $(\mathrm{P}=0.0085)$, both of which were positively associated with tuberculosis rates. In multiple regression analysis the only variable independently associated with tuberculosis was the proportion of households with more than 1.5 people per room. The regression parameter estimate was 2.63 (SE 0.85).

For the Asian population, no single variable was significantly associated with tuberculosis-for example, the proportion of households with more than 1.5 people per room had a regression coefficient of 0.433 (SE $0.313, \mathrm{P}=0.18$ ). The only covariate approaching significance was the proportion of households not owning their own home, which was marginally positively associated (regression parameter estimate 0.186, SE 0.098, $\mathrm{P}=0.067$ ). 


\section{Discussion}

We found significant associations between markers of deprivation and incidence of tuberculosis for the white population but not for the Asian population. This implies that the association between poverty and tuberculosis previously demonstrated ${ }^{45}$ may not be generalisable to non-white ethnic groups, which now account for more than half of cases of tuberculosis in the United Kingdom. ${ }^{3}$

Our study suggests two possible explanations for the association with poverty found by the earlier studies which did not separate ethnic groups on both predictor (deprivation) and outcome (tuberculosis) variables. The first is that the relation found at the whole population level (confirmed by our study) may simply reflect the relation between poverty and tuberculosis in the white population. Both the Liverpool ${ }^{4}$ and Leeds ${ }^{5}$ studies were carried out in populations that are likely to be predominantly white: Liverpool has a relatively small ethnic minority population, especially of south Asians, ${ }^{4}$ and ethnic minorities are likely to be under-represented in the elderly people studied in Leeds. This explanation is consistent with the finding that increasing deprivation has a greater effect on tuberculosis rates in areas with small immigrant populations than in areas with more immigrants. ${ }^{14}$

The second possible explanation is that markers of deprivation and the proportion of the population of Asian origin are confounded at the ecological level because ethnic minorities tend to live in poorer areas. These variables therefore are impossible to disentangle without using data on ethnic group. This situation is certainly true for Birmingham (fig 3) and London. ${ }^{15}$ The explanation is consistent with findings in two ecological studies which looked at trends in tuberculosis: a study of all districts in England and Wales found that whereas $29 \%$ of cases occurred in $10 \%$ of districts with the highest Jarman score, 33\% occurred in the districts with the highest proportion of ethnic minority residents, ${ }^{16}$ and a study of London boroughs found significant associations with both proportion of migrants and overcrowding but no independent association with unemployment and social class. ${ }^{17}$

\section{Potential biases and confounders}

Caution is needed when drawing conclusions about causation from ecological studies as they may not properly reflect association at the individual level because of confounding or modification of effect. ${ }^{18}$ In addition, regression analyses depend on the estimates of tuberculosis rates in each ward, and although this study contains four times as many cases as that in Liverpool and 15 times as many as that in Leeds, some of the ethnic specific ward rates are based on small numbers of cases. The precision of the rates is likely to depend on population size. We addressed this problem by weighting individual observations by population size, although an unweighted analysis produced similar results.

The lack of any association between poverty and tuberculosis in Asian people in our study is unlikely to be due to sample size (there were three times as many Asian patients as white ones) or insufficient variation between Asian populations in different wards. Although it is possible that the indices of deprivation used are not applicable between ethnic groups, it is
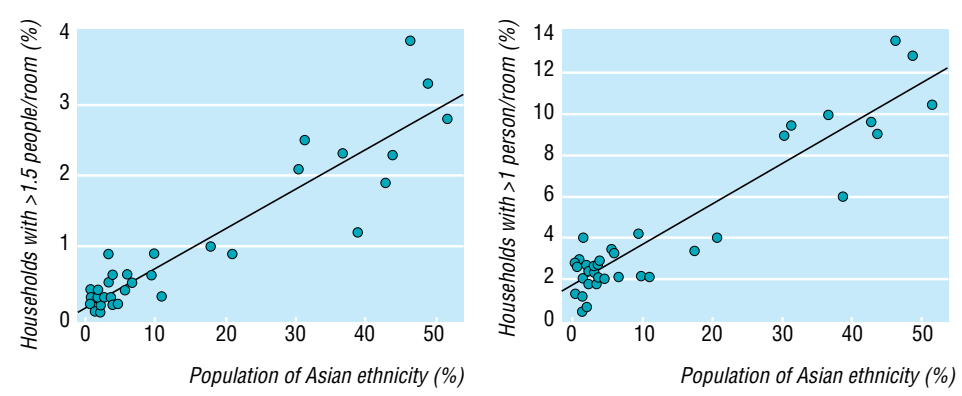

Fig 3 Relation between overcrowding and percentage of resident population of Asian ethnicity. Each point represents one electoral ward

unlikely that none of the 10 indices we used are valid proxies for deprivation in the Asian population. It therefore seems likely that other factors explain the variations in tuberculosis rates in this group. We suggest that the likelihood of close contact with an infectious case of tuberculosis is the key factor. This is supported by the observation that $20 \%$ of tuberculosis cases occurred within 12 months after a visit to the Indian subcontinent. ${ }^{7}$ Indeed, the ability to afford such a visit may have a confounding effect on the relation between poverty and tuberculosis.

The finding on multivariable analysis that overcrowding was the only factor associated with tuberculosis rates in white people independently of other variables was unexpected. If tuberculosis in white people is usually reactivation of latent infection then the mechanism of a cause and effect relation with overcrowding is not obvious. It may be that interactions between variables make multiple regression at the ecological level unreliable or that overcrowding is a marker for another factor that we could not measure, such as diet or education.

\section{Implications}

Our results support the conclusion that social factors, such as poverty, which influence the likelihood of developing (predominantly reactivated) tuberculosis in white people are likely to be different from those influencing the risk of contracting (predominantly new infection) tuberculosis in the Asian population. ${ }^{19}$

\section{Key messages}

- Previous studies in the United Kingdom have been unable to disentangle the effects of poverty and ethnicity on the incidence of tuberculosis

- A strong relation was found between measures of poverty and tuberculosis in the white population

- No relation between measures of poverty and tuberculosis was found in the Asian population

- The only measure of poverty independently associated with tuberculosis in the white population is overcrowding

- An increasing proportion of tuberculosis is occurring in Asian people and causal factors in this group are likely to be different from those in the white population 
Further research is necessary, including a case-control study to disentangle the confounding effects of poverty, ethnicity, and age at the individual level. In addition, recent developments in DNA analysis of Mycobacterium tuberculosis ${ }^{20}$ could be used to study patterns of transmission within and between ethnic groups. In the meantime, as national notification rates are 25 times higher in Asians than white people (and this inequality is widening), ${ }^{3}$ prevention of new infection in Asians by education, ${ }^{21}$ immunisation, ${ }^{22}$ and prompt diagnosis and treatment of infectious cases ${ }^{23}$ must remain a priority.

We thank Dr John Innes and the staff at Birmingham chest clinic for their work in maintaining the Birmingham tuberculosis register.

Contributors: JIH contributed to the formulation of the primary study hypothesis and led the design of the study, interpretation of the results, and writing of the paper. SSB contributed to formulation of the hypothesis, interpretation of the data, and writing the paper. SA collected all the data and contributed to the analysis of the data and writing of the paper. CPF led the analysis of the data and contributed to the design of the study, interpretation of results, and writing the paper. $\mathrm{JIH}$ will act as guarantor.

Funding: None.

Competing interests: None declared.

1 Weber HW. On prevention of tuberculosis. Tuberculosis 1899;1:101-11.

2 British Tuberculosis Association. Tuberculosis among immigrants to England and Wales: a national survey in 1965. A report from the research committee of the British Tuberculosis Association. Tubercle 1966;47:145-56. committee of the British Tuberculosis Association. Tubercle 1966;47:145-56.
Ormerod P. Tuberculosis and immigration. Br J Hosp Med 1996;56:209-12.

3 Ormerod P. Tuberculosis and immigration. Br J Hosp Med 1996;56:209-12. poverty. BMJ 1993;307:759-61.

Kearney MT, Warklyn PD, Teale C, Goldman JM, Pearson SB. Tuberculosis and poverty. BMJ 1993;307:1143.
6 Darbyshire JH. Tuberculosis: old reasons for new increase? Socioeconomic deprivation threatens tuberculosis control. BMJ 1995;310:954-5.

7 McCarthy OR. Asian immigrant tuberculosis - the effect of visiting Asia. Br J Dis Chest 1984;78:248-53.

8 Springett VH. Tuberculosis notification rates in the elderly. Common Dis Rep CDR Rev 1991;1:R149-50.

9 Medical Research Council Cardiothoracic Epidemiology Group. National survey of notifications of tuberculosis in England and Wales in 1988. Thorax 1992;47:770-5.

10 Vynnycky E, Fine PEM. The natural history of tuberculosis: the implications of age-dependent risks of disease and the role of re-infection. Epidemiol Infect 1997;119:183-201.

11 Townsend P, Phillimore P, Beattie A. Health and deprivation: inequalities in the North. London: Croon Helm, 1988.

12 Box GEP, Cox DR. An analysis of transformations. I $R$ Stat Soc 1964:26B:211-52.

13 Statistical Sciences. S-PLUS for windows user's manual, version 4. Seattle: Statistical Sciences, 1997.

14 Tocque K, Doherty MJ, Bellis MA, Spence DPS, Williams CDS, Davies PDO. Tuberculosis notifications in England: the relative effects of deprivation and immigration. Int J Tuberc Lung Dis 1998;2:213-8.

15 Doherty MJ, Davies PDO, Bellis MA, Tocque K. Ethnic origin is more important than social deprivation. BMJ 1995;311:187.

16 Bhatti N, Law MR, Morris JK, Halliday R, Moore-Gillon J. Increasing incidence of tuberculosis in England and Wales: a study of the likely causes. BMJ 1995;310:967-9

17 Mangtani P, Jolley DJ, Watson JM, Rodrigues LC. Socioeconomic deprivation and notification rates for tuberculosis in London during 1982-91. BMJ 1995;310:963-6.

18 Greenland S, Morgenstern H. Ecological bias, confounding, and effect modification. Int J Epidemiol 1989;18:269-74.

19 Bakhshi SS, Hawker JI, Ali S. The epidemiology of tuberculosis of ethnic group in Birmingham and its implications for future trends in tuberculosis in the UK. Ethnicity and Health 1997;2:147-53.

20 Borgdorff MW, Nagelkerke N, van Soolingen D, de Haas PEW, Veen J, van Embden JDA. Analysis of tuberculosis transmission in between nationalities in the Netherlands in the period 1993-1995 using DNA fingerprinting. Am J Epidemiol 1998;147:187-95.

21 Bakhshi SS, Ali S. Knowledge, attitude and behaviour of TB patients. J Public Health Med 1995;17:343-8.

22 Packe GE, Innes JA. Protective effect of BCG immunisation in infant Asians: a case control study. Arch Dis Child 1988;61:277-81.

23 World Health Organisation. Stop TB at the source. Report on the tuberculosis epidemic. Geneva: WHO, 1995.

(Accepted 9 June 1999)

\section{Department of Social Medicine, \\ University of \\ Bristol, Bristol \\ BS8 2PR \\ Elise Whitley \\ lecturer in medical \\ statistics \\ David Gunnell \\ senior lecturer in \\ epidemiology and \\ public health medicine \\ George Davey \\ Smith \\ professor of clinical \\ epidemiology \\ School of \\ Geographical \\ Sciences, University \\ of Bristol \\ Daniel Dorling \\ reader in geography \\ Correspondence to: \\ E Whitley \\ elise.whitley@ \\ bristol.ac.uk}

BMJ 1999;319:1034-7

\section{Abstract}

Objectives To investigate the association between suicide and area based measures of deprivation and social fragmentation.

Design Ecological study.

Setting 633 parliamentary constituencies of Great Britain as defined in 1991.

Main outcome measures Age and sex specific mortality rates for suicide and all other causes for 1981-92.

Results Mortality from suicide and all other causes increased with increasing Townsend deprivation score, social fragmentation score, and abstention from voting in all age and sex groups. Suicide mortality was most strongly related to social fragmentation, whereas deaths from other causes were more closely associated with Townsend score. Constituencies with absolute increases in social fragmentation and Townsend scores between 1981 and 1991 tended to have greater increases in suicide rates over the same period. The relation between change in social fragmentation and suicide was largely independent of Townsend score, whereas the association with Townsend score was generally reduced after adjustment for social fragmentation.

Conclusions Suicide rates are more strongly associated with measures of social fragmentation than with poverty at a constituency level.

\section{Introduction}

The association between deprivation and mortality is well established, and for most common diseases mortality is higher in people of lower socioeconomic position. Mortality also tends to be higher in populations living in poor areas, regardless of individual socioeconomic position. ${ }^{1}$ High risk areas have been identified by using census derived indices of poverty $^{2}$ and voting patterns. ${ }^{3}$ Although strong associations have been found between these area based indices and mortality, ${ }^{45}$ they may not fully describe the relation with area of residence for all causes of death.

Geographical studies indicate that people living in deprived areas generally have high suicide rates. ${ }^{67}$ Other analyses suggest that the proportion of single 